\title{
Identification of Novel EYS Mutations by Targeted Sequencing Analysis
}

\author{
Wanli Tian, ${ }^{1, *}$ Xiao Li, ${ }^{1, *}$ Ya Li, ${ }^{2, *}$ Luyao Wang, ${ }^{3}$ Yeming Yang, ${ }^{1}$ Kuanxiang Sun, \\ Wenjing Liu, ${ }^{1}$ Bo Zhou, ${ }^{3}$ Bo Lei, ${ }^{2}$ and Xianjun Zhu,
}

Purpose: Retinitis pigmentosa (RP) is an inherited and progressive degenerative retinal disease that often results in severe vision loss and blindness. However, mutations in known RP disease genes account for only $60 \%$ of RP cases, indicating that there are additional pathogenic mutations are yet to be identified. We aimed to identify the causative mutations in the eyes shut homolog $(E Y S)$ gene in a cohort of Chinese RP and rod-cone dystrophy families.

Materials and Methods: Targeted next-generation sequencing was applied to identify novel mutations in these patients. Candidate variants were evaluated using bioinformatics tools. Mutations were confirmed by Sanger sequencing.

Results: We identified eight heterozygous mutations in the EYS gene in the four probands, including a novel frameshift deletion mutation, c.8242_8243del (p.L2748fs); a novel insertion mutation, c.5802_5803insT (p.I1935YfsX6); a novel splicing mutation, c.1300-1G $>$ A; two heterozygous stop-gain mutations, c.1750G $>$ T (p.E584X) and c.8805C $>$ A (p.Y2935X); and three novel missense mutations, c.8269G $>$ A (p.V2757I), c. 2545C $>$ T (p.R849C) and c.7506C $>$ A (p.S2502R). Only c.8805C $>$ A had been reported previously in RP patients. None of these mutations were present in 1000 control individuals.

Conclusions: We identified seven novel mutations in the EYS gene, expanding the mutational specra of EYS in Chinese patients with RP and rod-cone dystrophy.

Keywords: NGS, EYS mutation, autosomal-recessive retinitis pigmentosa

\section{Introduction}

$\mathbf{R}$ ETINitis PIGMentosa (RP, OMIM\# 268000) refers to progressive degeneration of the retina, which exhibits genetic heterogeneity and a complex clinical phenotype (Daiger et al., 2013). Unfortunately, the pathogenesis of RP has not been completely elucidated (Pagon, 1988; Yang et al., 2020). RP is characterized by degeneration of rod cells, cone cells, and retinal pigment epithelial cells and by progressive functional impairment of the outer retina (Hamel, 2007). Patients with RP first develop night blindness and gradual loss of vision, and the disease progresses to classical fundus manifestations, such as bone spicule-like pigmentation of the retina (Tsujikawa et al., 2008; Khan et al., 2015). RP has a global prevalence of 1 in 3500 to 4000 individuals and affects $\sim 1$ million individuals (Hartong et al., 2006; Chen et al., 2014).
RP can be inherited in autosomal-dominant (30-40\%), autosomal-recessive (arRP, 50-60\%), or X-linked (5-15\%) patterns (Allard, 1983; Maria et al., 2015). In addition, the absence of a family history precludes the determination of its mode of inheritance, a condition known as sporadic RP (sRP). RP-related genes encode proteins that participate in visual signal transduction, the rhodopsin cycle, photoreceptor structure, and transcriptional regulation of photoreceptor cells. Mutations in these genes influence multiple biochemical pathways, including RNA intron splicing (Zhou et al., 2002; Maita et al., 2004), structural or cytoskeletal function (Gu et al., 1998; Clarke et al., 2000; Liu et al., 2004), synaptic or cell-cell interaction (Chapple et al., 2003; Reiners et al., 2005), and vitamin A metabolism (Xue et al., 2004; Moiseyev et al., 2005). More than 90 genes have been identified to be associated with

\footnotetext{
${ }^{1}$ The Sichuan Provincial Key Laboratory for Human Disease Gene Study, Department of Laboratory Medicine, Sichuan Provincial People's Hospital, School of Medicine, University of Electronic Science and Technology of China, Chengdu, China.

${ }^{2}$ People's Hospital of Zhengzhou University, Henan Provincial People's Hospital, Henan Eye Institute, Henan Eye Hospital, Zhengzhou, China.

${ }^{3}$ Psychosomatic Medicine Center, Sichuan Academy of Medicine and Sichuan Provincial People's Hospital, Chengdu, China.

${ }^{4}$ Department of Ophthalmology, First People's Hospital of Shangqiu, Shangqiu, China.

*These authors contributed equally to this work.
} 
RP and account for $\sim 60 \%$ of RP cases (RetNet website) (Zhang et al., 2018).

Eyes shut homolog (EYS, OMIM\# 612424) is one of the largest genes expressed in the retina. EYS was first identified as the gene causing arRP, located at the RP25 locus (6q12) and spanning a genomic region of more than $2 \mathrm{Mb}$ on chromosome 6 (Abd El-Aziz et al., 2008). The human EYS gene encodes an extracellular protein of 3165 amino acid residues, which starts with a signal peptide and contains 28 epidermal growth factor (EGF)-like domains followed by 5 LamG domains (Collin et al., 2008). EYS was reported to interact with matriglycan of $O$-mannosyl glycans by its LamG domains (Liu et al., 2020). The EYS protein is proposed to play a role in the modeling of retinal architecture in the human eye and required for ommatidium development in Drosophila (Husain et al., 2006; Zelhof et al., 2006). In zebrafish, EYS protein is localized to the connecting cilium/transition zone of photoreceptors and ablation of eys in zebrafish leads to mislocalization of outer segment proteins, F-actin disruption, and cone-rod dystrophy (Yu et al., 2016). Mutations in EYS have also been identified in cone-rod dystrophy patients (Littink et al., 2010; Katagiri et al., 2014; Sengillo et al., 2018; Pierrache et al., 2019) in addition to patients with RP. $E Y S$-associated retinal dystrophy is a common inheritable retinal dystrophy (IRD) in European and Japanese populations (Abd El-Aziz et al., 2010; Audo et al., 2010; Barragán et al., 2010; Arai et al., 2015; Chen et al., 2015; Maeda et al., 2018). EYS mutations account for $15.9 \%$ of arRP cases in a Spanish population (Barragán et al., 2010). A high prevalence (18-23\%) of $E Y S$-associated RP was reported in Japanese RP patients (Arai et al., 2015; Yang et al., 2020). In Chinese RP patients, the p.C2139Y mutation affecting the EGF-like domain of the EYS protein is a hotspot mutation (Gu et al., 2016; Xiao et al., 2019). However, the relationships between EYS variants and accurate clinical phenotypes are still uncertain because of the lack of large cohort studies in Chinese populations.

Next-generation sequencing (NGS), a recently emerging technique, has the advantages of large-scale, rapid, and systematic identification of variants. NGS approaches generally include targeted capture array sequencing, whole-exome sequencing, and whole-genome sequencing. Compared with traditional approaches for screening potential genes such as positional cloning and gene identification, NGS provides a novel and efficient technique for molecular diagnosis of RP.

In this study, we utilized a targeted NGS approach to assess causative mutations underlying the cases in a cohort of arRP and rod-cone dystrophy families from central and southwest China and identified eight compound heterozygous mutations in the EYS gene: c.8242_8243del (p.L2748fs), c.8269G $>A$ (p.V2757I), c.2545C $>$ T (p.R849C), c.5802_5803insT (p.I1935YfsX6), c.7506C >A (p.S2502R), c.1750G $>$ T (p.E584X), c.8805C >A (p.Y2935X), and c.1300$1 \mathrm{G}>$ A. Except for c.8805C $>$ A (p.Y2935X), all these mutations were novel mutations not reported previously. Our findings expanded the EYS mutation spectrum in Chinese patients with arRP and rod-cone dystrophy.

\section{Materials and Methods}

\section{Patient recruitment and ethics statement}

This study adhered to the tenets of the Declaration of Helsinki and was approved by the Institutional Ethics Review Boards of Sichuan Provincial People's Hospital and Henan Eye Institute. Written informed consent was obtained from all patients and their family members. All experiments were performed following the approved protocols.

\section{Genetic analysis by targeted NGS}

Genomic DNA was extracted from peripheral blood samples obtained from RP patients, unaffected relatives, and control individuals with DNA extraction kits (TianGen, Beijing, China) according to the manufacturer's instructions. DNA samples were subjected to targeted NGS analysis according to the previously described panel (Wang et al., 2013). In brief, DNA extracts were sheared into $250-500 \mathrm{bp}$ segments, and the sheared fragments were end-repaired with polynucleotide kinase. An A base was added to the $3^{\prime}$ ends with Klenow exonuclease. Finally, Illumina Y-shaped index

Table 1. Primers and Polymerase Chain Reaction Conditions Used in This Study

\begin{tabular}{|c|c|c|c|}
\hline Amplicon & Sequence $\left(5^{\prime}\right.$ to $\left.3^{\prime}\right)$ & Annealing temperature $\left({ }^{\circ} \mathrm{C}\right)$ & Products (bp) \\
\hline c. $1300-1 \mathrm{G}>\mathrm{A}$ & F-AGCCCATCAAACAGAAGATTAG & 58 & 362 \\
\hline c. $1750 \mathrm{G}>\mathrm{T}$ & $\begin{array}{l}\text { F-TGGATGCAGTTGTTTGAGTGA } \\
\text { R-TCTTCCCTCCTTTTATTGTGCT }\end{array}$ & 58 & 372 \\
\hline c. $2545 \mathrm{C}>\mathrm{T}$ & $\begin{array}{l}\text { F-TGGATGGACTGGACAGAACT } \\
\text { R-GTCCCCTACCCACAATGTACA }\end{array}$ & 62 & 374 \\
\hline c.5802-5803insT & $\begin{array}{l}\text { F-AAGAGGCAGGAAAGAGACGT } \\
\text { R-AATACTTGGTTGTTGTTGGTTC }\end{array}$ & 60 & 495 \\
\hline c. $7506 \mathrm{C}>\mathrm{A}$ & $\begin{array}{l}\text { F-TGGGGCTGGGAATAACAACA } \\
\text { R-GGCATCATGGGCTATTCACC }\end{array}$ & 60 & 438 \\
\hline c.8242_8243del & $\begin{array}{l}\text { F-TGAGAACTGTCCACAACTAGAG } \\
\text { R-CCTTCTGCACCAACTCTTCC }\end{array}$ & 62 & 386 \\
\hline c. $8269 \mathrm{G}>\mathrm{A}$ & $\begin{array}{l}\text { F-TGAGAACTGTCCACAACTAGAG } \\
\text { R-CCTTCTGCACCAACTCTTCC }\end{array}$ & 62 & 386 \\
\hline c. $8805 \mathrm{C}>\mathrm{A}$ & $\begin{array}{l}\text { F-AGGTGGCTCAAATGTAGGTGA } \\
\text { R-TGGAGACCAATTGCCAGAAA }\end{array}$ & 61 & 444 \\
\hline
\end{tabular}



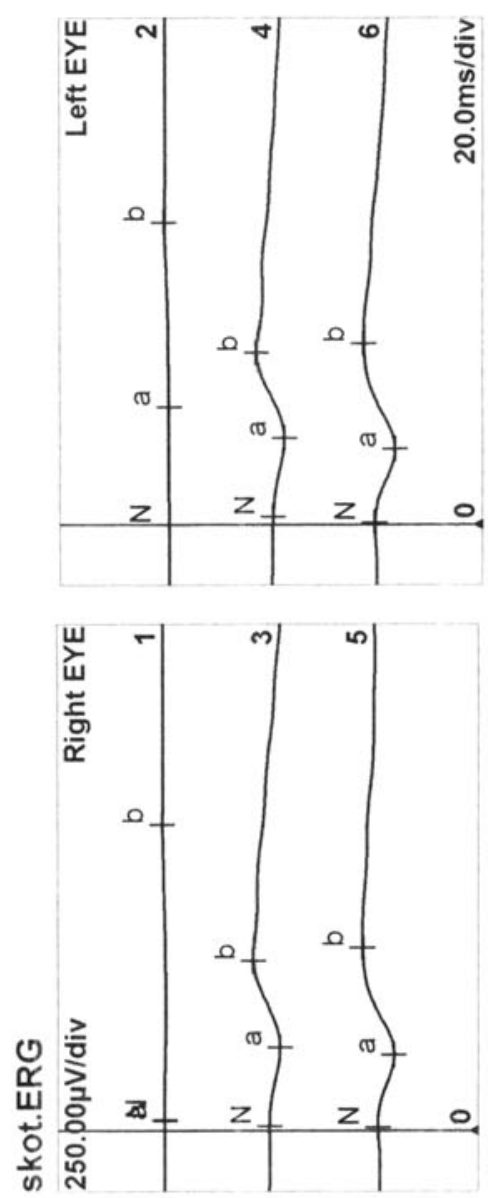

0
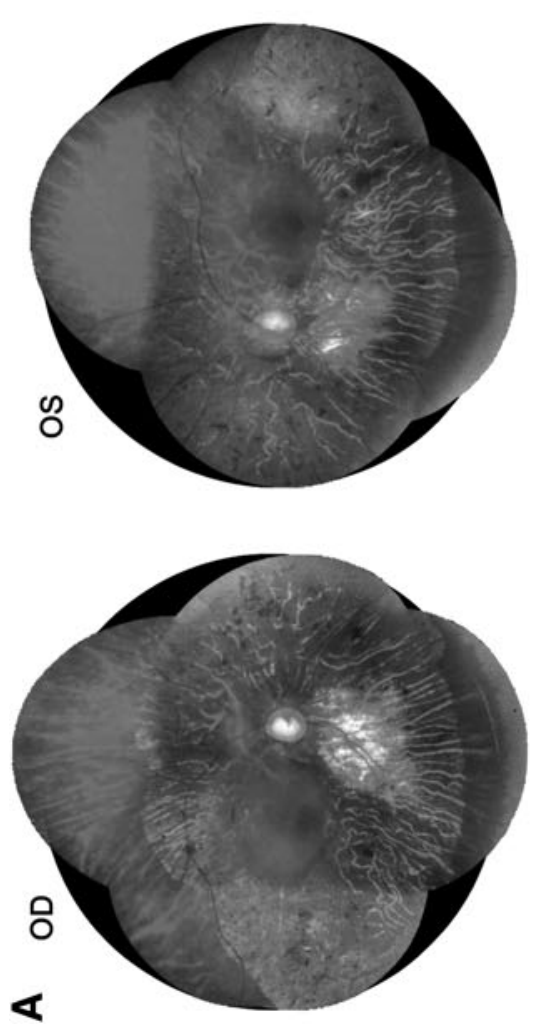
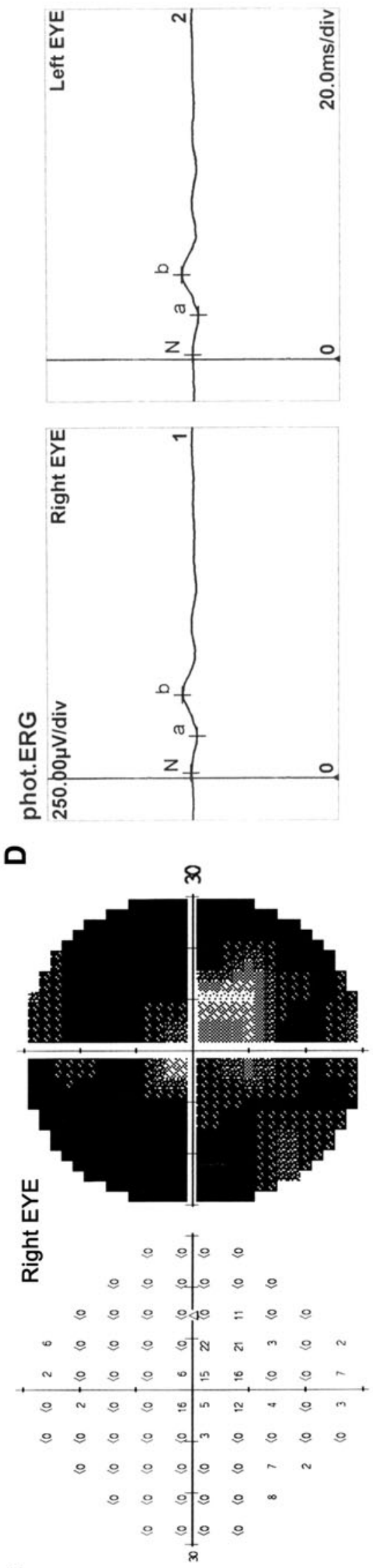

$\boldsymbol{m}$
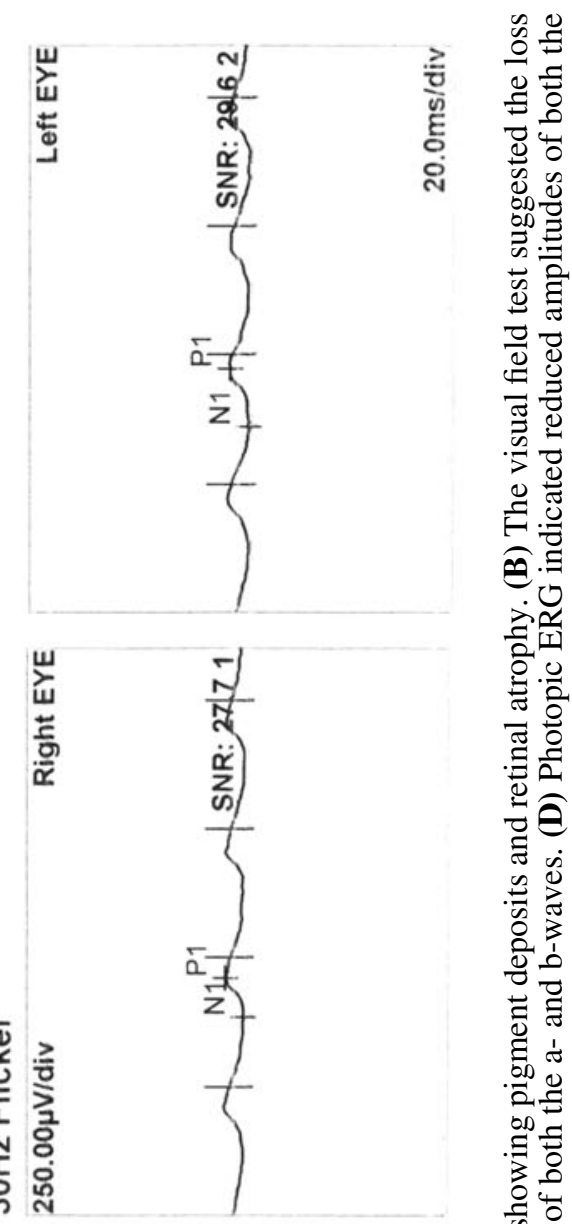

ш

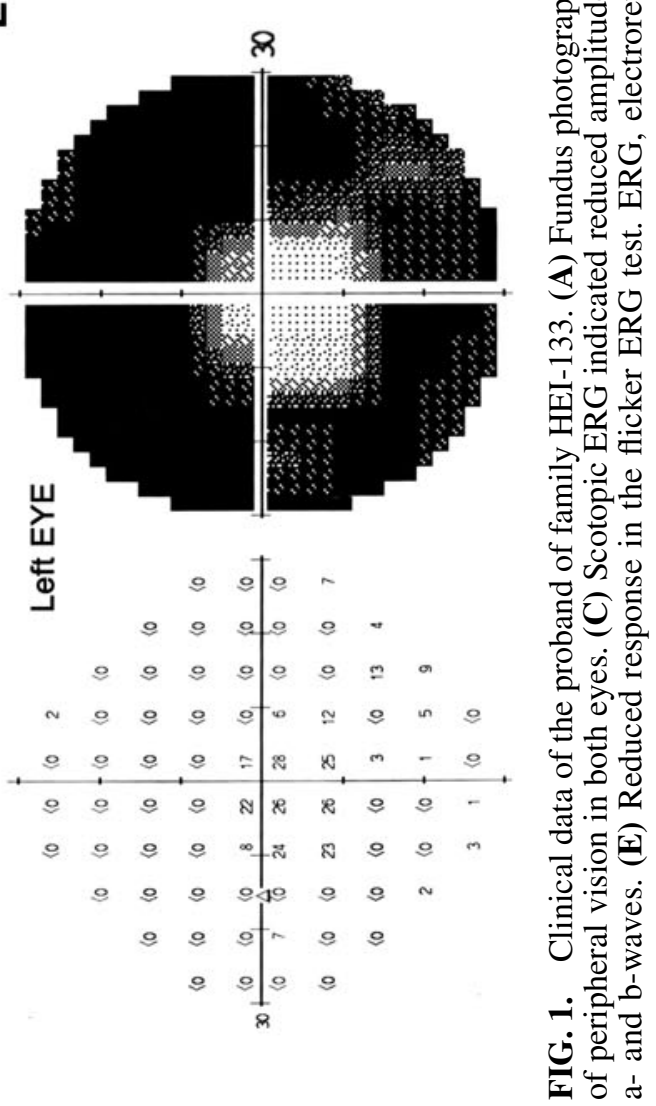


A

OS

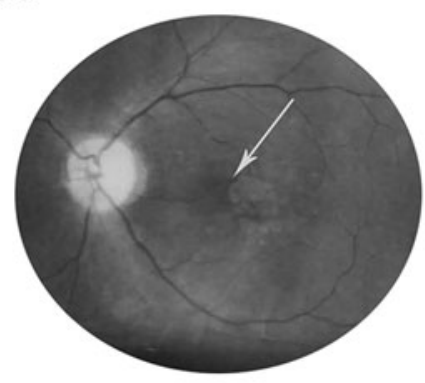

C

OS

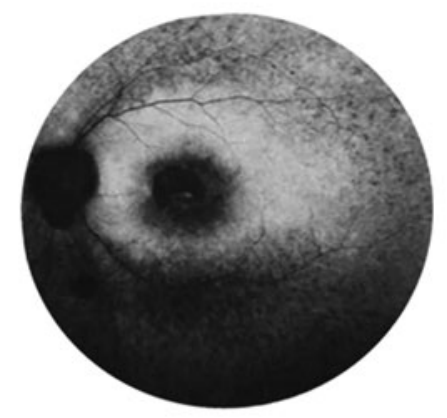

OD

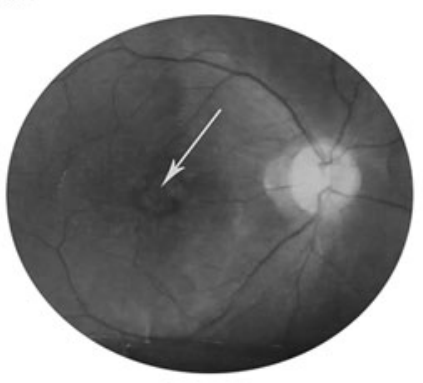

D

OD

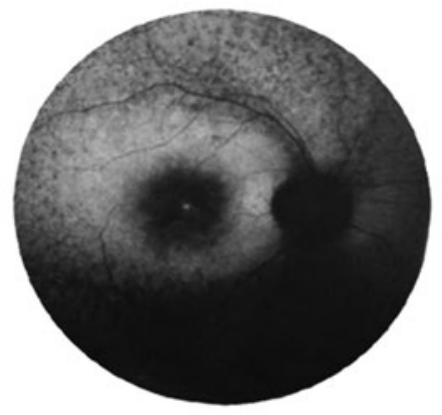

B
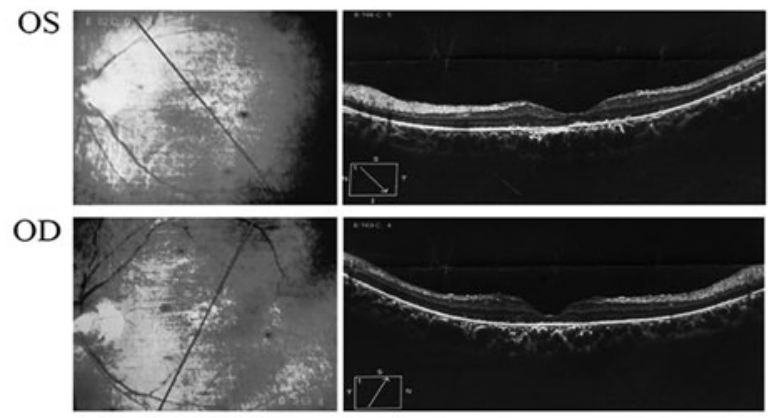

OS

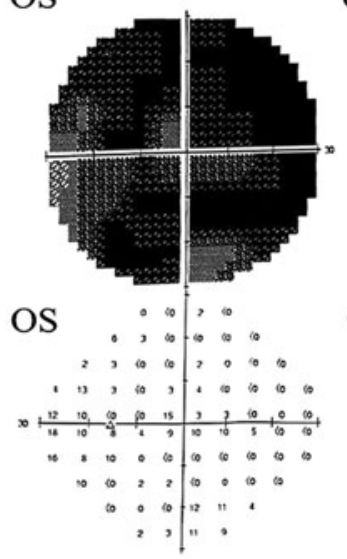

OD

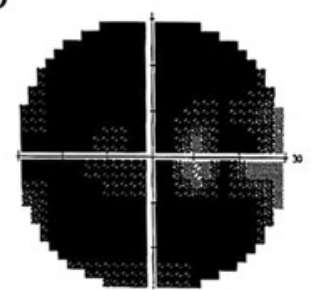

OD

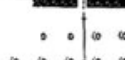

$+0.100$

(i)

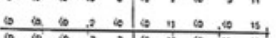

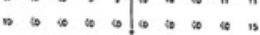

$00-0<0$

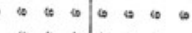

FIG. 2. Clinical data of the proband of family SCRP-18. (A) Fundus photograph showing deficiency of pigmented epithelial tissue with narrowed arterioles and pigment deposition. The arrows indicate macular regions. (B) OCT revealed disorganized photoreceptor layers. (C) Autofluorescence imaging of both eyes showed slightly increased fluorescence. The macular region exhibited small diffuse regions of high fluorescence, and the vascular arch exhibited pigment proliferation and fluorescence shadowing. (D) The visual field test suggested the loss of both peripheral and central vision in both eyes. OCT, optical coherence tomography.

adapters were used to construct paired-end DNA libraries, and the captured libraries were sequenced in an Illumina HiSeq 2000 sequencer (Illumina, Inc., San Diego, CA).

Sequencing reads were aligned to the reference human genome assembly hg38 UCSC using the Burrows-Wheeler Aligner (BWA) program. The "mpileup" command in SAMTOOLS v.0.1.19 was applied to identify insertions/deletions and single nucleotide variants. To filter and annotate the detected variants, three databases were referenced: NCBI CCDS, RefSeq, and Ensembl. The following exclusion steps were applied: Variants within intergenic, intronic, and untranslated regions and synonymous mutations were excluded. In addition, benign variants were eliminated with the online prediction tools PolyPhen-2 and SIFT. Mutations identified in our study were verified in the HGMD database to confirm whether the pathogenic mutations had been previously reported.

\section{Mutation verification by Sanger sequencing}

Sanger sequencing was carried out to confirm the potential mutations in the RP families. Polymerase chain reaction (PCR) primers (Table 1) were designed and synthesized by
Sangon Biotech (Shanghai, China) to amplify DNA fragments flanking the mutation site. PCR products were purified with FastAP Thermosensitive Alkaline Phosphatase (Thermo Scientific Fermentas, Waltham, MA) and were then sequenced in an ABI 3730 automated sequencer (Applied Biosystems, Foster City, CA).

\section{Results}

\section{Clinical assessments}

Targeted NGS analysis was performed on two Chinese arRP families and one rod-cone dystrophy family from the central and southwest regions of China. The proband of family HEI-133 was a 61-year-old man who exhibited night blindness, peripheral vision loss, and a reduced electroretinogram response (Fig. 1). The proband of family SCRP18 III: 1 was a 32-year-old man who experienced night blindness, progressive peripheral vision loss, optic disc atrophy, and retinal vascular stenosis (Fig. 2). Fundus images showed slight pigment deposition (Fig. 2A). Optical coherence tomography revealed disorganized photoreceptor and ellipsoid layers (Fig. 2B). Autofluorescence imaging of both eyes showed hypofluorescence in the macular region, a 


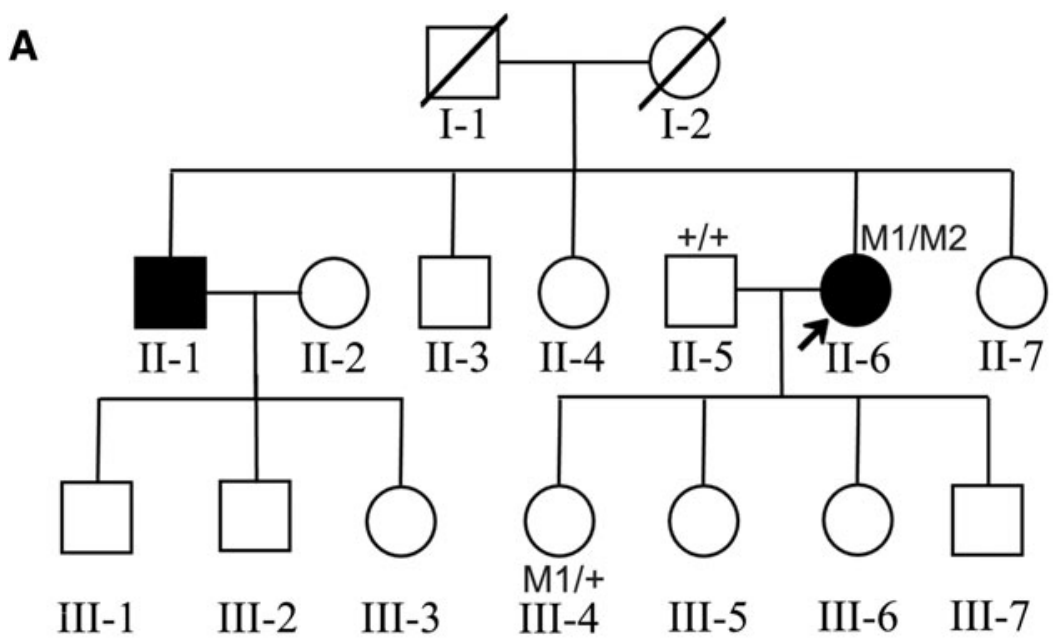

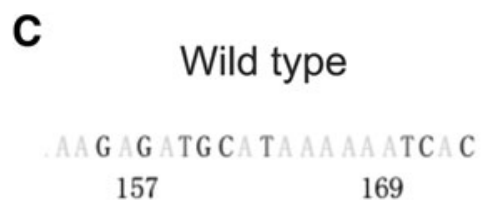

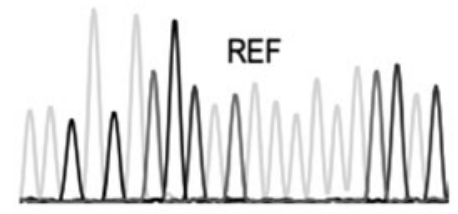

E

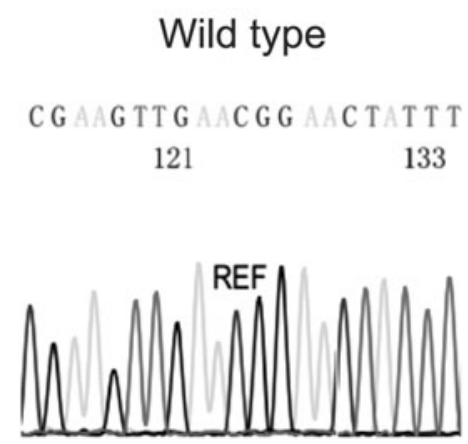

FIG. 3. Pedigree of the HEI-133 family and corresponding sequencing chromatograms. (A-E) Pedigree chart and EYS mutations identified in the HEI-133 family. The proband, II-6, carries the compound heterozygous mutations c.8243_8244del (M1) and c. $8269 \mathrm{G}>\mathrm{A}(\mathrm{M} 2)$. M1/M2 indicates individuals with compound heterozygous mutations; M1/+ indicates a heterozygous carrier; and $+/+$ indicates an individual carrying two wild-type alleles. Arrow indicates the affected proband. DEL AA indicates deletion of the AA dinucleotide; REF indicates the reference sequence. EYS, eyes shut homolog.
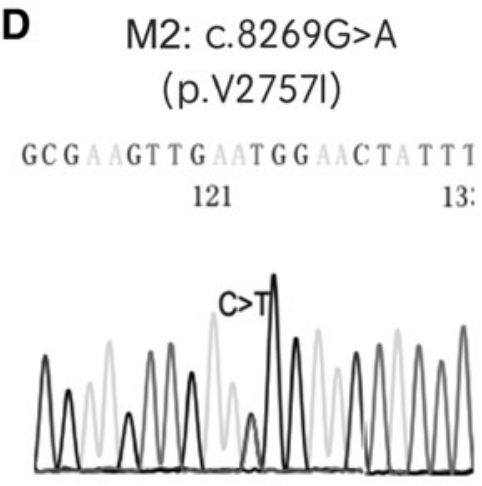

(p.R849C, M3) and the heterozygous insertion frameshift mutation c.5802_5803insT (p.I1935YfsX6, M4) were detected in the proband III:1 of family SCRP-18 (Fig. 4A). To validate these two mutations, we next collected DNA samples from the proband's parents for Sanger sequencing. The proband's father carried the c.2545C $>\mathrm{T}$ (p.R849C) mutation, whereas his mother carried the c.5802_5803insT (p.I1935YfsX6) mutation. His affected brother III:3 also carried the compound heterozygous mutations M3/M4. Unaffected brother III:2 carried heterozygous M4 allele and unaffected sister III:3 carried heterozygous M3 allele. Insertion of a $\mathrm{T}$ nucleotide led to a reading frameshift mutation and a truncated protein. In family arRP-30 (Fig. 4B), the heterozygous missense mutation c.7506C $>$ A (p.S2502R, M5) and the heterozygous stop-gain mutation c.1750G $>\mathrm{T}$ cleotide resulted in a frameshift mutation and a truncated protein. Moreover, the heterozygous mutation c. $2545 \mathrm{C}>\mathrm{T}$
The frameshift deletion mutation c.8242_8243del (p.L2748fs, M1) and the heterozygous mutation c.8269G $>$ A (p.V2757I, M2) in the EYS gene were identified in the pro- 


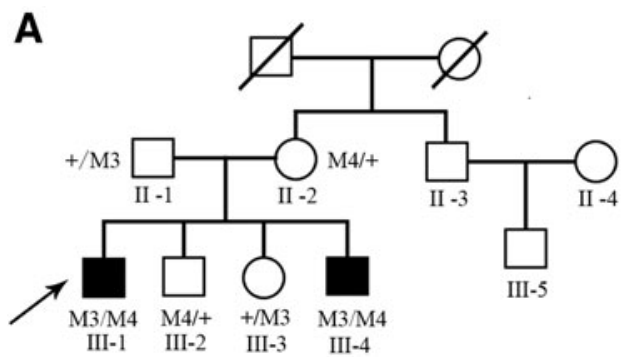

Family SCRP-18

B

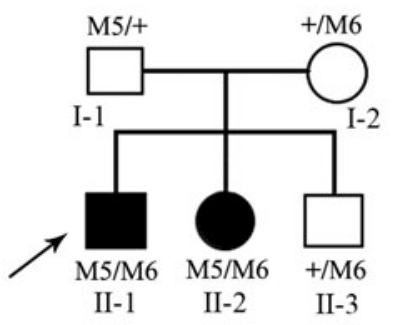

Family arRP-30

C

Sporadic arRP-35
C C A C C A A C G C T A T A

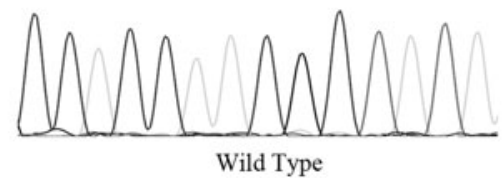

C C A C C A A C/T G C T A T A

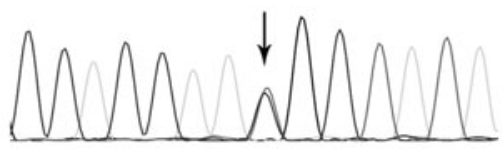

M3: c. $2545 \mathrm{C}>\mathrm{T}$ p.R849C

C A G G A G C A G C C C C

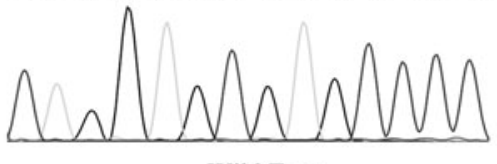

Wild Type

C A G G A G C/A G A G C C C C

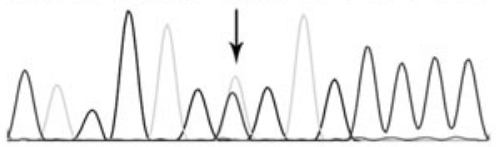

M5: c.7506C $>$ A p.S2502R.

T T C T T A C A G T T G C C

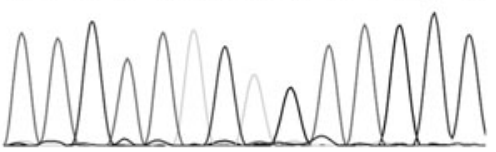

Wild Type

T T C T T A C/AAG T T G C C

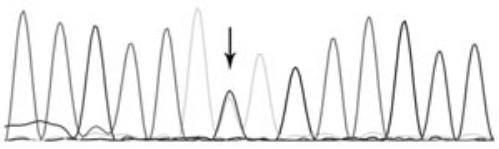

M7: c. 8505 C $>$ A p.E2935X
T T T T T T A T T C A A T T G

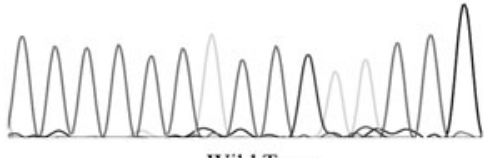

Wild Type

T T T T T T A T T C A A T T G $\downarrow$ TA T T C A A T T

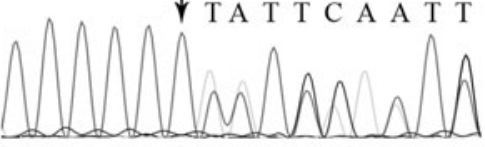

M4: c.5802-5803insT p.I1935YfsX6

A A A G A T G A A A T T A A

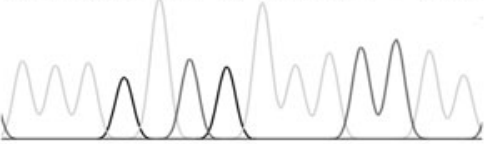

Wild Type

A A A G A T G/T A A A $T$ T A A

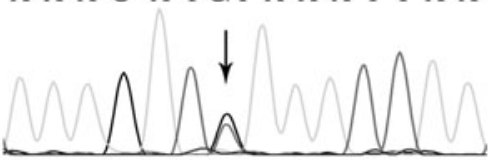

M6: c.1750G >T p.E584X

T T T G T A G T A T G T A T

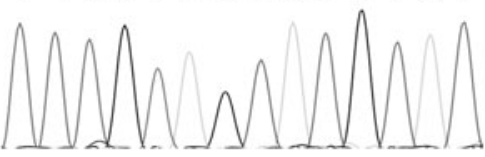

Wild Type

T T T G T A A/G TA T G T A T

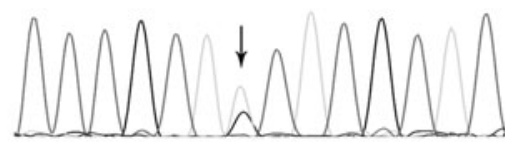

M8: c. $1300-1 \mathrm{G}>\mathrm{A}$

FIG. 4. Pedigrees of the SCRP-18 and aRP-30 families and the corresponding sequencing chromatograms. (A) Pedigree chart and EYS mutation identified in the SCRP-18 family. The proband, III-1, carries the compound heterozygous mutations c.2545C $>$ T (M3) and c.5802_5803insT (M4). M3/M4 indicates individuals with compound heterozygous mutations. His father II:1 carries M3/+ allele and his mother carries +/M4 allele. Arrow indicates the affected proband. His affected brother III:3 also carried the compound heterozygous mutations M3/M4. Unaffected brother III:2 carries heterozygous M4 allele and unaffected sister III:3 carries heterozygous M3 allele. (B) Pedigree chart and EYS mutations identified in the family arRP-30. The proband, II-1 carries the compound heterozygous mutations c.7506C $>$ A (M5) and c.1750G $>$ T (M6). M3/M4 indicates individuals carrying both mutations as compound heterozygous mutations. His father II:1 carried M5/+ allele and his mother carried +/M6 allele. M5/+ and +/M6 indicate heterozygous carriers. His affected sister II:2 also carried the compound heterozygous mutations M5/M6. Unaffected brother II:3 carried heterozygous M6 allele. Arrow indicates the affected proband. (C) The C.8805C >A (M7) mutation and c.1300-1G >A (M8) mutations in EYS were identified in a patient with sporadic arRP-35. Arrows indicate the altered nucleotides.

(p.E584X, M6) were identified in EYS. Sanger sequencing analysis confirmed that the proband's father carried the c.7506C $>$ A (p.S2502R, M5) mutation and that his mother carried the c.1750G $>$ T (p.E584X, M6) mutation. His affected sister II:2 also carried the compound heterozygous mutations M5/M6. Unaffected brother II:3 carried heterozygous M6 allele. The c.1750G $>$ T (p.E584X, M6) mutation is a nonsense mutation that results in premature termination of EYS protein translation (Fig. 5A).
In addition, in 12 patients with sRP in family arRP-35, we found 2 compound heterozygous mutations in the EYS gene (Fig. 4C). Specifically, the known nonsense mutation c. 8805C $>$ A (p.Y2935X, M7) and the novel splicing mutation c.1300-1G $>$ A (M8) in EYS were identified. The splicing mutation c.1300-1G>A (M8) led to the production of a truncated protein (Fig. 5A).

These 7 novel heterozygous mutations had not been previously reported in patients with RP and rod-cone dystrophy and 
A Exon

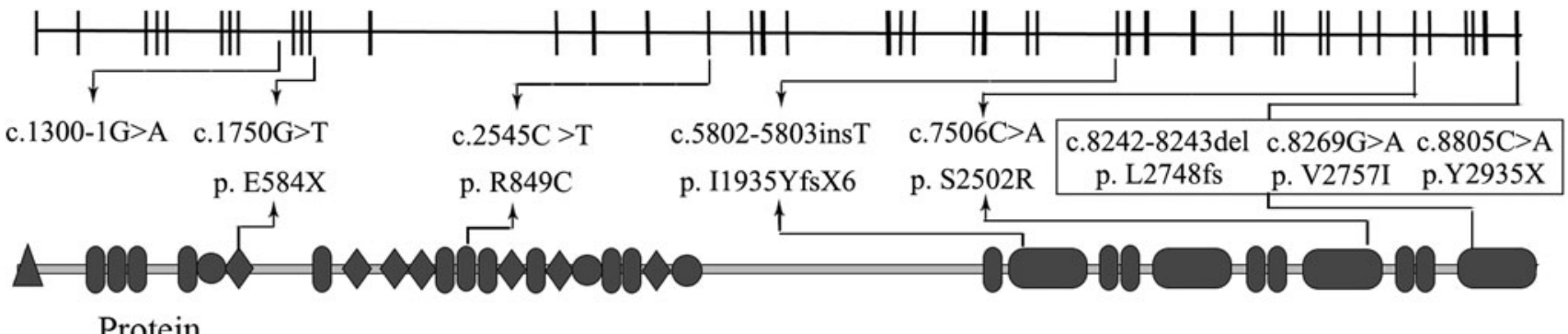

Protein

signal peptide $\triangle$ EGF $\quad$ cbEGF EGF like domain

Lamin G-like

B

H. sapiens
M. fascicularis
P. abelii
F. catus
C. lupus
V. vulpes
D. rerio

M. fascicularis

P.abelii

F.catus

C. lupus

V.vulpes

D. rerio
P.R849C

GQFCHQRYNLCDL

GQFCHQRYNPCDL

GRFCHQRYNPCDL

- - - - - - - - -

GKFCHQHYNPCDC

GKFCHQHYNPCDC

GPLCEQPYDPCEL

\section{P. Y2935X}

PDQSFSYSCLCTL

PDQSFSYSCLCTL

PNQSFSYSCLCTL

PDQSFSYNCFCAL

PDQ - FSYSCLCAL

PDQ - FSYSCLCAL

NSTSASYSCMCSL

\begin{abstract}
P.I1935YfsX6
LVDGFFIQLFIEN

LVDGFFIQLSIEN

LVDGFFIQLFIEN

SVDEFFIQLFIEN

SVDKFFTQLFVGN

SVDGFLIQLFIEN

KRSVFFIKLYISN
\end{abstract}

\begin{abstract}
P.E584X
EAVCKDEINRPRC

EAICEDEINRPRC

EAICKDEINRPRC

EAIYKDEINRPRC

EVLYKDEINKSRC

EVLYKDEINKSRC

QAVCQDLVNGFQC
\end{abstract}

P.S2502R

GIASIRSEPLNLS
GIASIRSDPLNLS
GIASIRSEPLNLS
GTASISSDPLDLS
GMASISSDPLDLS
GMASISSDPLDLS
GLATIISDRLNPR

P.L2748fS P.V2757I
QHLKAQSGDFLCISLVNSSVQLRYNLGDRT
QHLKAQSGDFLCISLVNSSVQLRYNLGDRT
QHLKAQSGDFLCISLVNGSVQLRYNLGDRT
QHLKAQSGDFLCISLVNGSVQLRYNLGDRT
QHLKAQSGDFLCISLVNGFVQLRYNLGDRT
QHLKAQSGDFLCISLVNGFVQLRYNLGDRT
QHLSTHSGDFLSISLSAGFLQLRYNLGNQT

FIG. 5. EYS mutations in protein domains and sequence alignment of the affected amino acid residues. (A) Distribution of different mutations across the various domains of the EYS protein. (B) Comparison of the EYS amino acid sequence across homologous genes in various species. C. lupus, Canis lupus; D. rerio, Danio rerio; F. catus, Felis catus; H. sapiens, Homo sapiens; P. abelii, Pan abelii; V. vulpes, Vulpes vulpes.

were not present in our in-house database of 1000 ethnicitymatched control samples. Those seven amino acid residues affected by these seven mutations identified in this study are highly conserved in vertebrates, indicating the importance of these residues (Fig. 5B). In addition, the effects of these mutations were predicted with the online tools PolyPhen- 2 or SIFT, and the frequencies of these mutations were analyzed in the Genome Aggregation Database (gnomAD) (Table 2). Ac- cording to the American College of Medical Genetics and Genomics (ACMG) guidelines, the three frameshift mutations (p.L2748fs, p.I1935YfsX6, and p.E584X) and one splicing mutation (c.1300-1G $>$ A) identified in this study are likely causative mutations. The three missense mutations (p.V2757I, p.R849C and p.S2502R) should be classified as variants of uncertain significance. In summary, our data expand the mutation spectrum of EYS in the Chinese population.

Table 2. Summary of Pathogenic Variants in EYS

\begin{tabular}{|c|c|c|c|c|c|c|}
\hline Pedigree & $\begin{array}{c}\text { Nucleotide } \\
\text { mutations }\end{array}$ & $\begin{array}{c}\text { Amino acid } \\
\text { changes }\end{array}$ & $\begin{array}{l}\text { Frequencies } \\
\text { in gnom } A D\end{array}$ & SIFT $($ cutoff $=0.05)$ & $\begin{array}{c}\text { PolyPhen-2 } \\
(\text { cutoff }=1)\end{array}$ & $D b S N P-I D s$ \\
\hline \multirow[t]{2}{*}{ HEI-133 } & c.8242_8243del & p.L2748fs & 0.000006722 & N/A & N/A & rs1240944758 \\
\hline & c. $8269 \mathrm{G}>\mathrm{A}$ & p.V2757I & 0.000006381 & Deleterious (0.04) & Deleterious (0.536) & rs775398034 \\
\hline \multirow[t]{2}{*}{ SCRP-18 } & c. $2545 \mathrm{C}>\mathrm{T}$ & p.R849C & 0.00005858 & Deleterious (0.03) & Deleterious (0.997) & rs369347845 \\
\hline & c.5802-5803insT & p.I1935YfsX6 & 0.000006997 & Deleterious $(0.01)$ & Deleterious (0.969) & rs 1455826633 \\
\hline \multirow[t]{2}{*}{ arRP-30 } & c. $1750 \mathrm{G}>\mathrm{T}$ & p.E584X & 0.000004003 & $\mathrm{~N} / \mathrm{A}$ & N/A & rs527236072 \\
\hline & c. $7506 \mathrm{C}>\mathrm{A}$ & p.S2502R & 0.0001648 & Neutral (0.07) & Deleterious (1.000) & rs77113519 \\
\hline \multirow[t]{2}{*}{ arRP-35 } & c. $8805 \mathrm{C}>\mathrm{A}$ & p.Y2935X & 0.00003719 & N/A & N/A & $\mathrm{N} / \mathrm{A}$ \\
\hline & c. $1300-1 \mathrm{G}>\mathrm{A}$ & N/A & 0.000003988 & N/A & N/A & $\mathrm{N} / \mathrm{A}$ \\
\hline
\end{tabular}

DbSNP, the single nucleotide polymorphism database; EYS, eyes shut homolog; N/A, not available. 


\section{Discussion}

Mutations in the EYS gene are recognized as a significant cause of arRP. EYS mutations were found to account for $15.9 \%$ of arRP cases in a Spanish population and $23 \%$ of arRP cases in Japanese patients (Pagon, 1988; Barragán et al., 2010). In this study, we used targeted NGS to analyze a cohort of Chinese arRP and rod-cone dystrophy families and identified eight heterozygous mutations in the EYS gene: c.8242_8243del, c. $8269 \mathrm{G}>\mathrm{A}, \quad$ c. $2545 \mathrm{C}>\mathrm{T}, \quad$ c.5802_5803ins $\mathrm{T}, \quad$ c.7506C $>\mathrm{A}$, c. $1750 \mathrm{G}>\mathrm{T}$, c. $8805 \mathrm{C}>\mathrm{A}$, and c.1300-1G $>$ A (Fig. 5). The proband of family SCRP-18 exhibited rod-cone dystrophy symptoms and carried c.2545C $>$ T and c.5802_5803insT. Similarly, Yang et al. (2020) reported rod-cone dystrophy patients carrying EYS mutations in a Japanese population (Pagon, 1988). Therefore, different mutations in EYS could cause distinct phenotypes in patients.

In this study, the identified c.5802_5803insT (p.I1935YfsX6) mutation resulted in a frameshift change at codon 1935 and a truncated protein, disrupting the Laminin G-like domain. The stop-gain mutation c. 1750G $>\mathrm{T}$ (p.E584X) disrupted translation at codon 584 and produced a truncated protein that lacked most of the calcium-binding EGF-like domain. The stop-gain mutation c. $8805 \mathrm{C}>\mathrm{A}$ (p.Y2935X), the missense mutation c.8269G $>$ A (p.V2757I), and the frameshift deletion mutation c.8242_8243del (p.L2748fs) were located in the same exon and EGF domain. The stop-gain mutation c. 8805C >A (p.Y2935X) was located at codon 2935, resulting in a truncated protein. The c. $2545 \mathrm{C}>\mathrm{T}$ (p.R849C) and c.7506C $>$ A (p.S2502R) mutations were located at codon 849 and codon 2502, respectively, and affected conserved amino acid residues (Fig. 5). Therefore, these identified mutations might be pathogenic mutations and could contribute to the development of photoreceptor degeneration.

In summary, our study identified five novel biallelic heterozygous EYS mutations in RP patients by a targeted NGS approach. In addition, two biallelic mutations in a rod-cone dystrophy patient were identified. Our study expands the mutation spectrum of the EYS gene in the Chinese population.

\section{Acknowledgments}

The funders played no role in the study design, data collection or analysis, decision to publish, or article preparation.

\section{Author Disclosure Statement}

No competing financial interests exist.

\section{Funding Information}

This work was supported by research grants from the Natural Science Foundation of China (81770950 to Xianjun Zhu and 81770949 to Bo Lei), Sichuan Department of Science and Technology (2020JDYH0027 to Xianjun Zhu), and Chinese Academy of Medical Sciences (2018PT31049 to Bo Zhou).

\section{References}

Abd El-Aziz MM, Barragan I, O’Driscoll CA, et al. (2008) EYS, encoding an ortholog of Drosophila spacemaker, is mutated in autosomal recessive retinitis pigmentosa. Nat Genet 40:1285-1287.

Abd El-Aziz MM, O’Driscoll CA, Kaye RS, et al. (2010) Identification of novel mutations in the ortholog of Droso- phila eyes shut gene (EYS) causing autosomal recessive retinitis pigmentosa. Invest Ophthalmol Vis Sci 51:42664272.

Allard RE (1983) Retinitis pigmentosa-an overview. J Am Optom Assoc 54:793-800.

Arai Y, Maeda A, Hirami Y, et al. (2015) Retinitis pigmentosa with EYS mutations is the most prevalent inherited retinal dystrophy in Japanese populations. J Ophthalmol 2015: 819760.

Audo I, Sahel JA, Mohand-Saïd S, et al. (2010) EYS is a major gene for rod-cone dystrophies in France. Hum Mutat 31: E1406-E1435.

Barragán I, Borrego S, Pieras JI, et al. (2010) Mutation spectrum of EYS in Spanish patients with autosomal recessive retinitis pigmentosa. Hum Mutat 31:E1772-E1800.

Chapple JP, Grayson C, Hardcastle AJ, et al. (2003) Organization on the plasma membrane of the retinitis pigmentosa protein RP2: investigation of association with detergentresistant membranes and polarized sorting. Biochem J 372: 427-433.

Chen X, Liu Y, Sheng X, et al. (2014) PRPF4 mutations cause autosomal dominant retinitis pigmentosa. Hum Mol Genet 23: 2926-2939.

Chen X, Liu X, Sheng X, et al. (2015) Targeted next-generation sequencing reveals novel EYS mutations in Chinese families with autosomal recessive retinitis pigmentosa. Sci Rep 5: 8927.

Clarke G, Goldberg AF, Vidgen D, et al. (2000) Rom-1 is required for rod photoreceptor viability and the regulation of disk morphogenesis. Nat Genet 25:67-73.

Collin RW, Littink KW, Klevering BJ, et al. (2008) Identification of a $2 \mathrm{Mb}$ human ortholog of Drosophila eyes shut/spacemaker that is mutated in patients with retinitis pigmentosa. Am J Hum Genet 83:594-603.

Daiger SP, Sullivan LS, Bowne SJ (2013) Genes and mutations causing retinitis pigmentosa. Clin Genet 84:132-141.

Gu S, Lennon A, Li Y, et al. (1998) Tubby-like protein-1 mutations in autosomal recessive retinitis pigmentosa. Lancet 351:1103-1104.

Gu S, Tian Y, Chen X, et al. (2016) Targeted next-generation sequencing extends the phenotypic and mutational spectrums for EYS mutations. Mol Vis 22:646-657.

Hamel CP (2007) Cone rod dystrophies. Orphanet J Rare Dis 2: 7.

Hartong DT, Berson EL, Dryja TP (2006) Retinitis pigmentosa. Lancet 368:1795-1809.

Husain N, Pellikka M, Hong H, et al. (2006) The agrin/perlecan-related protein eyes shut is essential for epithelial lumen formation in the Drosophila retina. Dev Cell 11: 483-493.

Katagiri S, Akahori M, Hayashi T, et al. (2014) Autosomal recessive cone-rod dystrophy associated with compound heterozygous mutations in the EYS gene. Doc Ophthalmol 128:211-217.

Khan SY, Ali S, Naeem MA, et al. (2015) Splice-site mutations identified in PDE6A responsible for retinitis pigmentosa in consanguineous Pakistani families. Mol Vis 21:871-882.

Littink KW, Koenekoop RK, van den Born LI, et al. (2010) Homozygosity mapping in patients with cone-rod dystrophy: novel mutations and clinical characterizations. Invest Ophthalmol Vis Sci 51:5943-5951.

Liu Q, Zuo J, Pierce EA (2004) The retinitis pigmentosa 1 protein is a photoreceptor microtubule-associated protein. J Neurosci 24:6427-6436. 
Liu Y, Yu M, Shang X, et al. (2020) Eyes shut homolog (EYS) interacts with matriglycan of O-mannosyl glycans whose deficiency results in EYS mislocalization and degeneration of photoreceptors. Sci Rep 10:7795.

Maeda A, Yoshida A, Kawai K, et al. (2018) Development of a molecular diagnostic test for retinitis pigmentosa in the Japanese population. Jpn J Ophthalmol 62:451-457.

Maita H, Kitaura H, Keen TJ, et al. (2004) PAP-1, the mutated gene underlying the RP9 form of dominant retinitis pigmentosa, is a splicing factor. Exp Cell Res 300:283-296.

Maria M, Ajmal M, Azam M, et al. (2015) Homozygosity mapping and targeted sanger sequencing reveal genetic defects underlying inherited retinal disease in families from pakistan. PLoS One 10:e0119806.

Moiseyev G, Chen Y, Takahashi Y, et al. (2005) RPE65 is the isomerohydrolase in the retinoid visual cycle. Proc Natl Acad Sci USA 102:12413-12418.

Pagon RA (1988) Retinitis pigmentosa. Surv Ophthalmol 33: 137-177.

Pierrache LHM, Messchaert M, Thiadens A, et al. (2019) Extending the spectrum of EYS-associated retinal disease to macular dystrophy. Invest Ophthalmol Vis Sci 60:2049-2063.

Reiners J, van Wijk E, Märker T, et al. (2005) Scaffold protein harmonin (USH1C) provides molecular links between Usher syndrome type 1 and type 2. Hum Mol Genet 14:3933-3943.

Sengillo JD, Lee W, Nagasaki T, et al. (2018) A distinct phenotype of eyes shut homolog (EYS)-retinitis pigmentosa is associated with variants near the C-terminus. Am J Ophthalmol 190:99-112.

Tsujikawa M, Wada Y, Sukegawa M, et al. (2008) Age at onset curves of retinitis pigmentosa. Arch Ophthalmol 126:337340.

Wang X, Wang H, Sun V, et al. (2013) Comprehensive molecular diagnosis of 179 Leber congenital amaurosis and juvenile retinitis pigmentosa patients by targeted next generation sequencing. J Med Genet 50:674-688.

Xiao X, Cao Y, Chen S, et al. (2019) Whole exome sequencing reveals novel EYS mutations in Chinese patients with autosomal recessive retinitis pigmentosa. Mol Vis 25:35-46.

Xue L, Gollapalli DR, Maiti P, et al. (2004) A palmitoylation switch mechanism in the regulation of the visual cycle. Cell 117:761-771.

Yang L, Fujinami K, Ueno S, et al. (2020) Genetic spectrum of EYS-associated retinal disease in a large Japanese cohort: identification of disease-associated variants with relatively high allele frequency. Sci Rep 10:5497.
Yu M, Liu Y, Li J, et al. (2016) Eyes shut homolog is required for maintaining the ciliary pocket and survival of photoreceptors in zebrafish. Biol Open 5:1662-1673.

Zelhof AC, Hardy RW, Becker A, et al. (2006) Transforming the architecture of compound eyes. Nature 443:696-699.

Zhang S, Li J, Li S, et al. (2018) Targeted next-generation sequencing reveals that a compound heterozygous mutation in phosphodiesterase $6 \mathrm{a}$ gene leads to retinitis pigmentosa in a Chinese family. Ophthalmic Genet 39:487-491.

Zhou Z, Licklider LJ, Gygi SP, et al. (2002) Comprehensive proteomic analysis of the human spliceosome. Nature 419: $182-185$.

Address correspondence to: Xianjun Zhu, PhD

The Sichuan Provincial Key Laboratory for Human Disease Gene Study

Department of Laboratory Medicine Sichuan Provincial People's Hospital School of Medicine

University of Electronic Science and Technology of China Chengdu 610072

China

E-mail: xjzhu@uestc.edu.cn

Bo Lei, MD, PhD

People's Hospital of Zhengzhou University Henan Provincial People's Hospital Henan Eye Institute Henan Eye Hospital Zhengzhou 450003

China

E-mail: bolei99@126.com

Bo Zhou, MD

Psychosomatic Medicine Center Sichuan Academy of Medicine and Sichuan Provincial People's Hospital Chengdu 610072

China

E-mail: tonyac7721@163.com 